\title{
Computational assessment of the structural analysis of different cyclooctene isomers
}

\author{
Joseph Lianbuanga*, Zodinpuia Pachuau, R. Lalthlamuana \\ Department of chemistry, Mizoram University, Tanhril 796004, Mizoram, India \\ Corresponding author: josephlbpachuau@yahoo.com
}

\begin{abstract}
Fifteen isomers of cyclooctene systems calculated in ab initio reveal the potency of computational results as a sufficient approach to distinguish different isomers and their configurational and conformational isomers within the system. The optimized geometries obtained from the semi empirical calculations were calculated to further optimized the isomers in Hartree-Fock (HF), density functional theory (DFT) and the perturbation theory of the second order (MP2) using 6-31G*, 6-311G* basis sets to locate the local minima. The geometrical parameters in support of the orientations and spatial arrangements of the structure in 3dimensional space of each isomer relatively substantiate their enthalpies of formation, respectively. The study enquires the stabilities of the isomers, where bicyclo[3.2.1]octane (Iso-I) is the most stable and 1,1dimethylbicyclopropane (Iso-XV) is the least stable. Optimized geometries of the configurational isomers have comparative influence the stability of the isomers.
\end{abstract}

KEYWORD: Cyclooctene, DFT, enthalpy of formations, isomers, geometrical analysis and stability.

\section{INTRODUCTION}

The cyclooctene $\left(\mathrm{C}_{8} \mathrm{H}_{14}\right)$ system is chosen for the study because of its wide applications. Not all the isomers do have applicability, and though the chemistry of some of the isomers are well known but the possibility of having varied isomers with some isomers bestowed with interesting applicability within the system cannot be ignored. Cyclo-octane is being used as hydrocarbon fuel and is used for increasing the specific impulse in liquid propellant rocket engine and rocket power unit for realizing the same (Ma et al., 2011).

Tetramethyl-cis-diaza-bicyclo [4.2.0] octane-3 is the backbone of pharmaceutical industry and 5-dione derivatives have differentiation, which includes activity and antiviral activity (Watson, 2010), and as such 7,7,8,8,tetramethyl-cis-diaza-bicyclo[4.2.0]octane-3,5,-dione derivatives with an activity to induce the differentiation of tumor cells are useful anti-tumor agent (Camps et al., 2010). The bicyclo [3.3.0] octane (octahydropentalene) has a rigid junction and the constrained aliphatic template is of interest with respect to the design of selfassembling motifs (Kendhale et al., 2008).

The study approached the $\mathrm{C}_{8} \mathrm{H}_{14}$ system with ten isomers modeled for computational calculations and grouped into four major structural differentiations as shown in Figure 1. The isomers studied in toto are fifteen due to the necessity of obtaining configurational and conformational isomers for selected simulated structures, in order to obtain maximum minimization of energy (local minima) along their respective potential energy surface. The isomers studied are bicyclo[3.2.1] octane (Iso-I), bicyclo[2.2.2]octane (Iso-II), cisoctahydropentalene (Iso-III), trans-octahydropentalene (Iso-IV), cis-bicyclo[4,2,0]octane (Iso-V), trans-bicyclo [4.2.0]octane (Iso-VI), cis-bicyclo[5.1.0]octane (Iso-VII), trans-bicyclo[5.1.0]octane (Iso-VIII), spiro[3.4]octane (Iso -IX), spiro[2.5]octane (Iso-X), trans-cyclopropylcyclopentane (Iso-XI), cis-cyclopropylcyclopentane (Iso-XII), trans-bicyclo butyl (Iso-XIII), cis-bicyclobutyl (Iso-XIV) and 1',1-dimethylbicyclopropane (Iso-XV) .

The isomers are of the types of the two general structural categories of bicyclic rings; the bridgedhead bicyclic rings, where two non-bonded atoms conjoint 3 rings, and the normal or fused bicyclic rings, where two bonded carbon atoms conjoint 2 rings as highlighted by black dots in the exemplary structures in Figure 2. The study also incorporates the spiro bicyclic rings where the two rings connects through a common atom shared by the 2 rings and in the isolated bicyclo rings, the two rings are joint through a $\mathrm{C}-\mathrm{C}$ bond arising from each end of the rings (also highlighted in Figure 2). 


\section{METHODOLOGY}

The levels of calculations employed were the minimal basis functions in semi empirical method (AM1), HartreeFock (HF), density functional theory (B3LYP) and the second order perturbation (MP2) with a split valence double -zeta polarized basis sets and split valence triple-zeta polarized basis sets of orbital approximations using Gaussian 98 package (Kozintsev, 1999). The geometries were optimized to obtain the local minima in attaining the selfconsistent field molecular orbital (SCF-MO) (Kumar, 2008) along the potential energy surface through a restricted closed-shell formalism and without any symmetry restrictions and vibrational analysis.

The semi-empirical AM1 (Austin model 1) treats only the valence electrons, and the molecular orbitals found are only rough approximations to the Hartree-FockRoothaan MOs. The molecular geometries and molecular dissociation energies calculated using this method is not very accurate though they give satisfactory computer costs. A more accurate result is obtained using the $a b$ initio methods of HF and MP2 with basis sets $6-31 \mathrm{G}(\mathrm{d})$ and $6-311 \mathrm{G}(\mathrm{d})$ respectively.

Using the same basis functions, the most widely used functional in DFT calculations is also employed using the hybrid functional called the B3LYP (Becke, Lee, Yang and Parr) with three empirical parameters, whose values were chosen to optimize the performance. The Local density approximation (LDA) of the density functional theory, based on electron density of molecule corrected both gradient and exchange corrections in the isomers imparting implicit structural parameters of bond length, bond angle and dihedral angle (Prasad, 1996) for the geometrical analysis.

\section{RESULTS AND DISCUSSION}

Fifteen isomers of $\mathrm{C}_{8} \mathrm{H}_{14}$ systems were simulated by investigating their enthalpies of formation to differentiate their stability from their total energies (in Hartress). Table 1 shows the enthalpies of formation at various levels of approximation methods using $6-31 G^{*}$ and 6311G* basis functions. The enthalpies of formation obtained are relatively close to each other for the density functional theory with both the basis of functions employed. With the exception of the semi empirical calculations, the ab initio Hartree-Fock and second order perturbation theory are in close agreement to their resultant calculations, and the approximate total energy differences with DFT is -2.32750 Hartrees for MP2 and 2.27780 Hartrees for HF. Since the calculated values obtained from B3LYP/6-311G* level gives the most stable data for all the isomers under quest, our interpretations of the results will be based from the resultant calculation of B3LYP/6-311G* level.

\section{Enthalpy of formation}

The enthalpy of formation for all the isomers are given in total energy (Hartrees) as shown in Table 1, and the relative energy (RE) are given in $\mathrm{kcal} / \mathrm{mol}$, where the most stable isomer is taken as $0 \mathrm{kcal} / \mathrm{mol}$ with respect to the total energy of the enthalpy of formation. Subsequently, the relative energies in $\mathrm{kcal} / \mathrm{mol}$ of the other isomers are being converted to determine their stabilities with respect to the most stable isomer. The most stable isomer studied, the bridgedhead isomer, Iso-I or bicyclo[3.2.1] octane is taken as $0 \mathrm{kcal} / \mathrm{mol}$. Iso-I is taken as a reference value for the conversion of all the other isomers in $\mathrm{kcal} / \mathrm{mol}$ (RE).

The bridgedhead bicyclic ring, Iso-I being the most stable isomer has a difference of $0.70 \mathrm{kcals} / \mathrm{mol}$ energy difference with the other bridgedhead isomer, Iso-II or bicyclo[2.2.2]octane which is the third most stable isomer. The normal or fused bicyclic ring configurational isomer, Iso-III or cis-octahydropentalene enthalpy of formation is the closest in enrgy to Iso-I, with a difference of only $0.24 \mathrm{kcal} / \mathrm{mol}$. The other bicyclic isomers of fused bicyclic rings are in the order of their configurational isomers as Iso-IV or trans-octahydropentalene, Iso-V or cisbicyclo[4,2,0]octane and Iso-VII or cisbicyclo[5.1.0]octane with $8.14 \mathrm{kcal} / \mathrm{mol}, 19.84 \mathrm{kcal} / \mathrm{mol}$ and $19.91 \mathrm{kcal} / \mathrm{mol}$, respectively. The configurational isomers for the cis geometries are seen to attain stability in comparison to their counterpart trans configurational isomers for the fused bicyclic rings. The spiro bicyclic rings subsequently follow the stability order as Iso-IX or spiro[3.4]octane and Iso-X or spiro[2.5]octane with $20.44 \mathrm{kcal} / \mathrm{mol}$ and 22.40 $\mathrm{kcal} / \mathrm{mol}$, respectively.

The isomers within the differentiation of Isolated bicyclic rings results in the stabilities of the trans isomeric counterpart, where both the trans isomers, Iso-XI or trans-cyclopropylcyclopentane and Iso-XIII or transbicyclo butyl are more stable in comparison to their counterpart conformational isomers, Iso-XII or ciscyclopropylcyclopentane and Iso-XIV or cis-bicyclobutyl, with their enthalpies of formation as $22.82 \mathrm{kcal} / \mathrm{mol}$ and $38.78 \mathrm{kcal} / \mathrm{mol}$, respectively. Two configurational isomers, Iso-VI or trans-bicyclo[4.2.0]octane and Iso-VIII or trans-bicyclo[5.1.0]octane from the fused bicyclic rings differentiation occupies positions of instability with 26.61 $\mathrm{kcal} / \mathrm{mol}$ and $32.02 \mathrm{kcal} / \mathrm{mol}$, respectively, just before the stability order of Iso-XIII and Iso-XIV. Finally, Iso-XV or 1,1 -dimethylbicyclopropane of the isolated bicyclic rings occupies the least stable position with $45.21 \mathrm{kcal} / \mathrm{mol}$ from out of the fifteen isomers studied along with their configurational and conformational isomers.

The stability order can be given as, Iso-I > cis Iso-III > Iso-II $>$ trans Iso-IV $>$ cis Iso- $V>$ cis Iso- $\mathrm{VII}>$ Iso-IX $>$ Iso- $\mathrm{X}$ $>$ trans Iso-XI $>$ cis Iso-XII $>$ trans Iso-VI $>$ trans Iso-VIII $>$ trans Iso-XIII > cis Iso-XIV > Iso-XV. The relative stabilities for the bicyclic rings differentiation can be rationalized from the enthalpies of formation, where the bridgedhead 


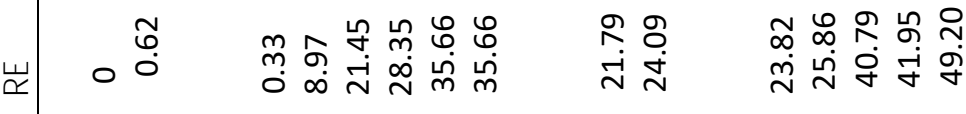

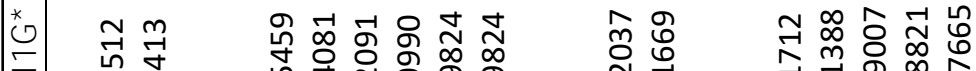

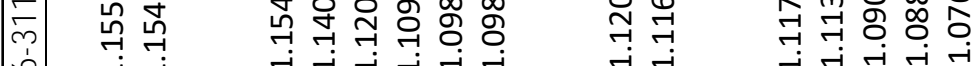

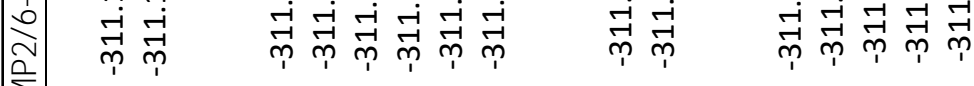

文

山্

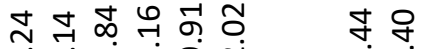

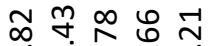

$\circ \infty$ gi

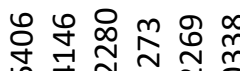

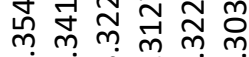

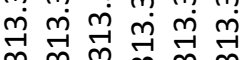

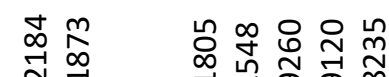
mm. $\vec{m} \quad \vec{m} \vec{m}$ ते

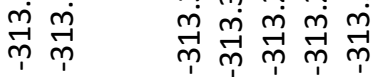

$\underset{\approx}{w} \quad 0 \stackrel{0}{0}$

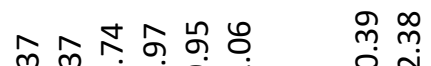

กั

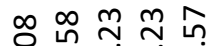

○ெ ำ ํำ

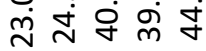

ㅇํㅇํํํํํㅇำ

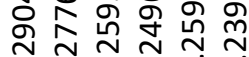
$\stackrel{m}{m} \stackrel{m}{m} \stackrel{m}{m} \stackrel{m}{m} \stackrel{m}{m} \stackrel{m}{m} \stackrel{m}{m}$

เี่

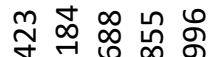

$\stackrel{\sim}{N} \quad \stackrel{\sim}{\sim} \stackrel{\sim}{N} \stackrel{\sim}{\sim}$

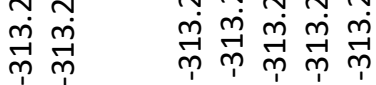

щᄄ

₹ $\stackrel{\infty}{\sim} \stackrel{n}{\sim}$ ํํำ

८ำ ○ें

$\stackrel{\text { ํ் }}{\sim}$

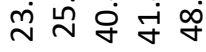

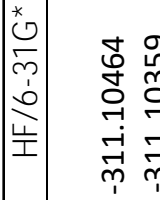

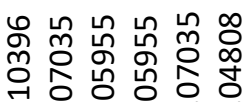

윙 융

ํํํㄱㄱㅇํำ

\& \& 0 \%

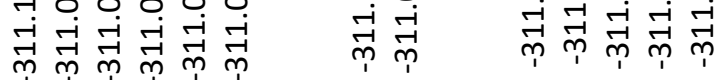

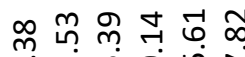

กิก นึก

은 웅 ชัฐ

穴

ஸ

\begin{tabular}{ll}
$\hat{\infty}$ & 0 \\
0 & \multirow{2}{n}{} \\
& 0 \\
0 & 0 \\
0 & 1
\end{tabular}

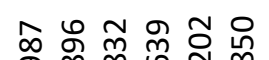

\%ั

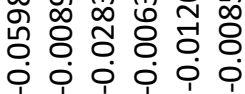

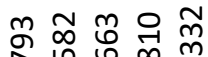

命

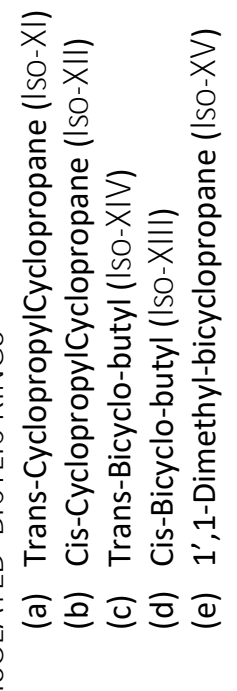


bicyclic rings are the most stable, followed by the fused bicyclic rings and the spiro bicyclic rings. The least stable are the isolated bicyclic rings. The stability order for the bicylic rings differentiation can be given as bridgedhead bicyclic rings > fused bicyclic rings > spiro bicyclic rings > isolated bicyclic rings. Judging from the enthalpies of formation and the resultant data, configurational and conformational geometries can be identified synonymous to the plausible geometrical strains and released of strains arising from the orientations and the projections of atoms or group of atoms in the ring system, thus predicting tremendous influence upon the stability of the isomers studied for $\mathrm{C}_{8} \mathrm{H}_{14}$ systems. The study observed that the cis-configurational isomers for all the fused bicyclic rings are more stable in comparison to their trans counterparts, whereas the trans conformational isomer for isolated bicyclic rings are more stable in comparison to their cis counterparts.

\section{Geometrical analysis}

The bond lengths, bond angles and dihedral angles were analyzed to predict the orientations of the isomers as shown in Table 2(a) to Table 2(d).

\section{Bridgedhead bicyclic rings}

As mentioned, Iso-I enthalpy of formation is taken as $0 \mathrm{kcals} / \mathrm{mol}$ to the total energy in Hartrees generated from the computational calculation, thus locating the isomer as the most stable for the system studied. The stability with reference to the geometrical parameters of bond lengths ranging between $1.54 \AA$ to $1.56 \AA$ relates typical $\mathrm{C}-\mathrm{C}$ bond and the bond angles ranging from $101.01^{\circ}$ to $111.88^{\circ}$, except for $33.75^{\circ}$ encountered within the 7-membered rings all falls within a tetrahedral C-C bond angles to comparative minimum distorted angles. Iso-I is a conjoint bridgedhead bicyclic ring of 3 rings of different sizes (5-membered, 6-membered and 7membered) with C-5 (Figure in Table 2(a)) taken as the bridgedhead. The distorted tetrahedral angle $\left(101.01^{\circ}\right)$ at the bridged head carbon along with its closely related distorted angle of $101.99^{\circ}$ for the 5-membered ring forms an envelope shape in the ring moiety, which may have cause strains in the angles of the ring. The other two conjoint rings, the 6-membered ring takes the form of the most stable chair conformer of cyclohexane rings and the 7-membered that of a tub shape indicating the release of angle strains in both the ring moiety to off-set the strain experienced by the 5 -membered conjoint ring. Though the angle strain is immense for $33.75^{\circ}$ at one end of the 7-membered ring, the ring size do compensate this strain and the overall picture of Iso-I and the angle strains present therein, especially for the 5-membered ring may have been compensated by the larger ring sizes and stable conformer from the other two conjoint rings the structure is endowed.

The dihedral angles established the projections of the three conjoint rings like a propeller shape. The stability of Iso-III over Iso-II would entail the release in angle strains for Iso-III from the normal tetrahedral to $114.93^{\circ}$ at the bonding carbons of the fused rings being joint together

TABLE 2(a): Geometrical structures of bridgedhead bicyclic rings showing the bond lengths $(\AA)$, bond angles $\left({ }^{\circ}\right)$ and dihedral angles $(\theta)$.

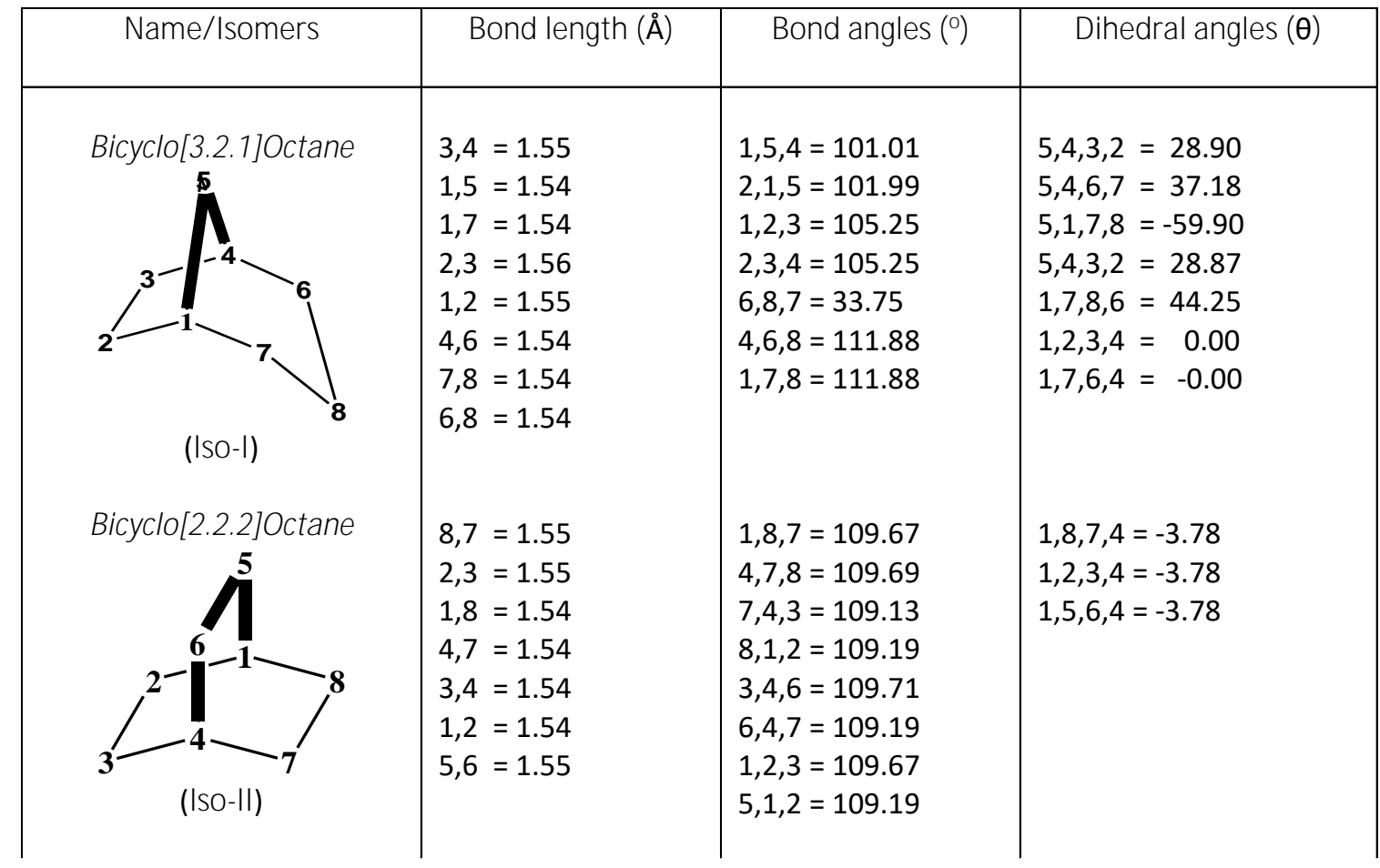


Bridgedhead Bicyclic Rings (Iso-I and Iso-II)
Normal or Fused Bicyclic Rings (Iso-III to Iso-VIII)

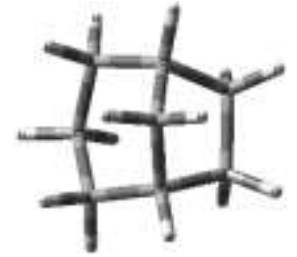

Iso-I

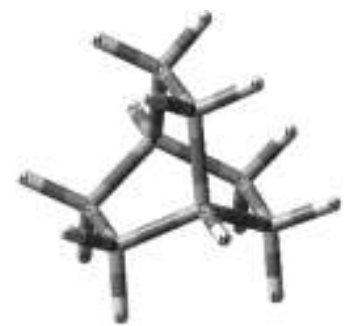

Iso-II

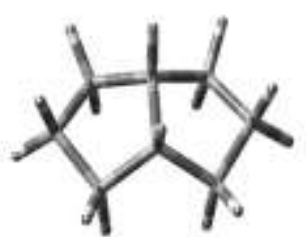

Iso-III

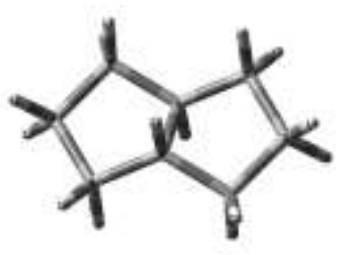

Iso-IV

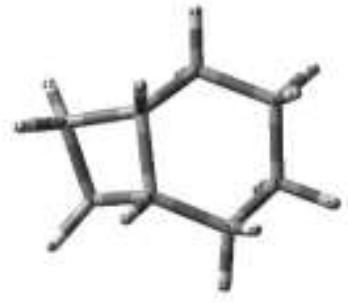

Iso-V

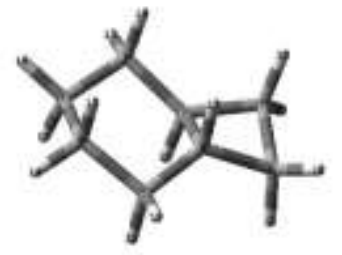

Iso-VI
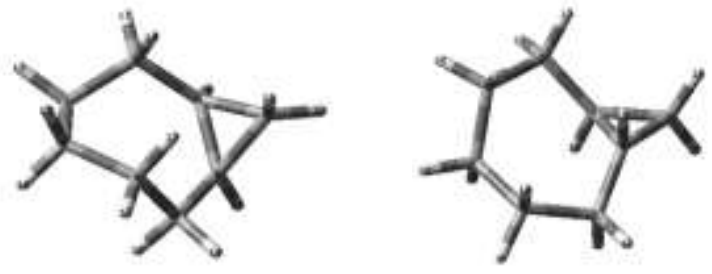

Spiro Bicyclic Rings (Iso-IX, Iso-X)

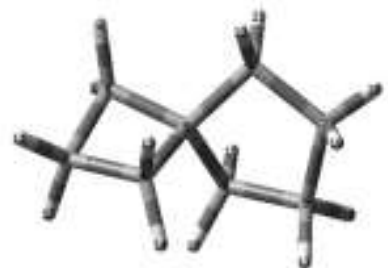

Iso-IX

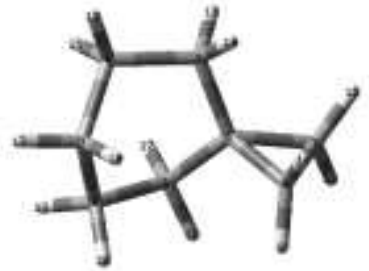

Iso-X

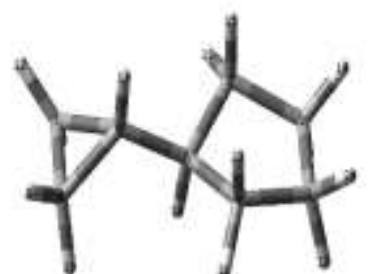

Iso-XI

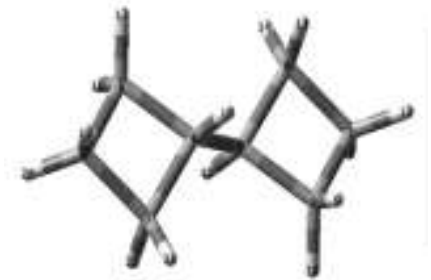

Iso - XIV

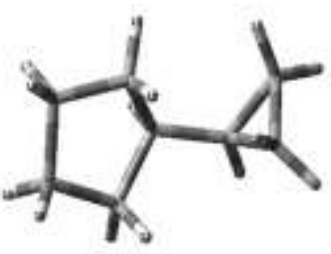

Iso-XIII

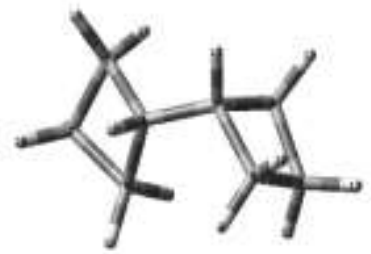

Iso-XII

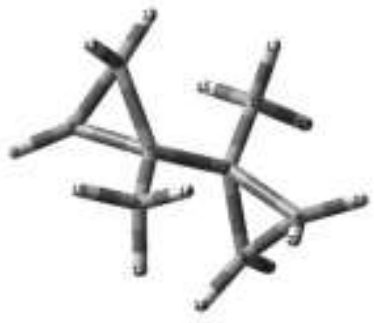

Iso-XV

Figure 1: Differentiation of bicyclic rings and the 10 isomers with their configurational isomers for $\mathrm{C}_{8} \mathrm{H}_{14} \mathrm{systems}_{\text {. }}$ 
TABLE 2(b): Geometrical structures of normal or fused bicyclic rings showing the bond lengths (angstroms $\AA$ ), bond angles (degrees ${ }^{\mathbf{0}}$ ) and dihedral angles $(\theta)$.

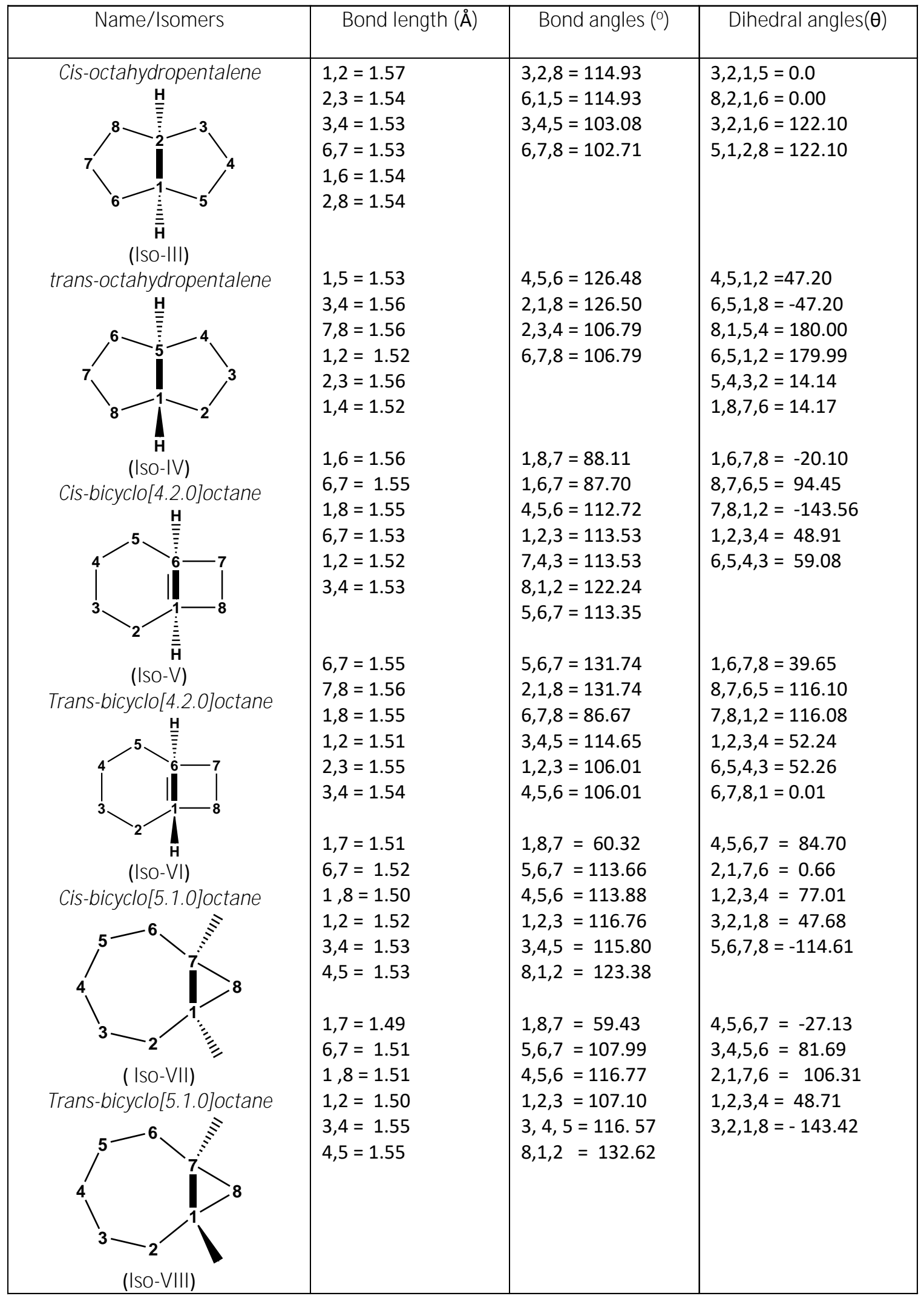


TABLE 2(c): Geometrical structures of spiro bicyclic rings showing the bond lengths (angstroms $\AA$ ), bond angles $\left(\right.$ degrees $^{\mathbf{o}}$ ) and dihedral angles $(\theta)$.

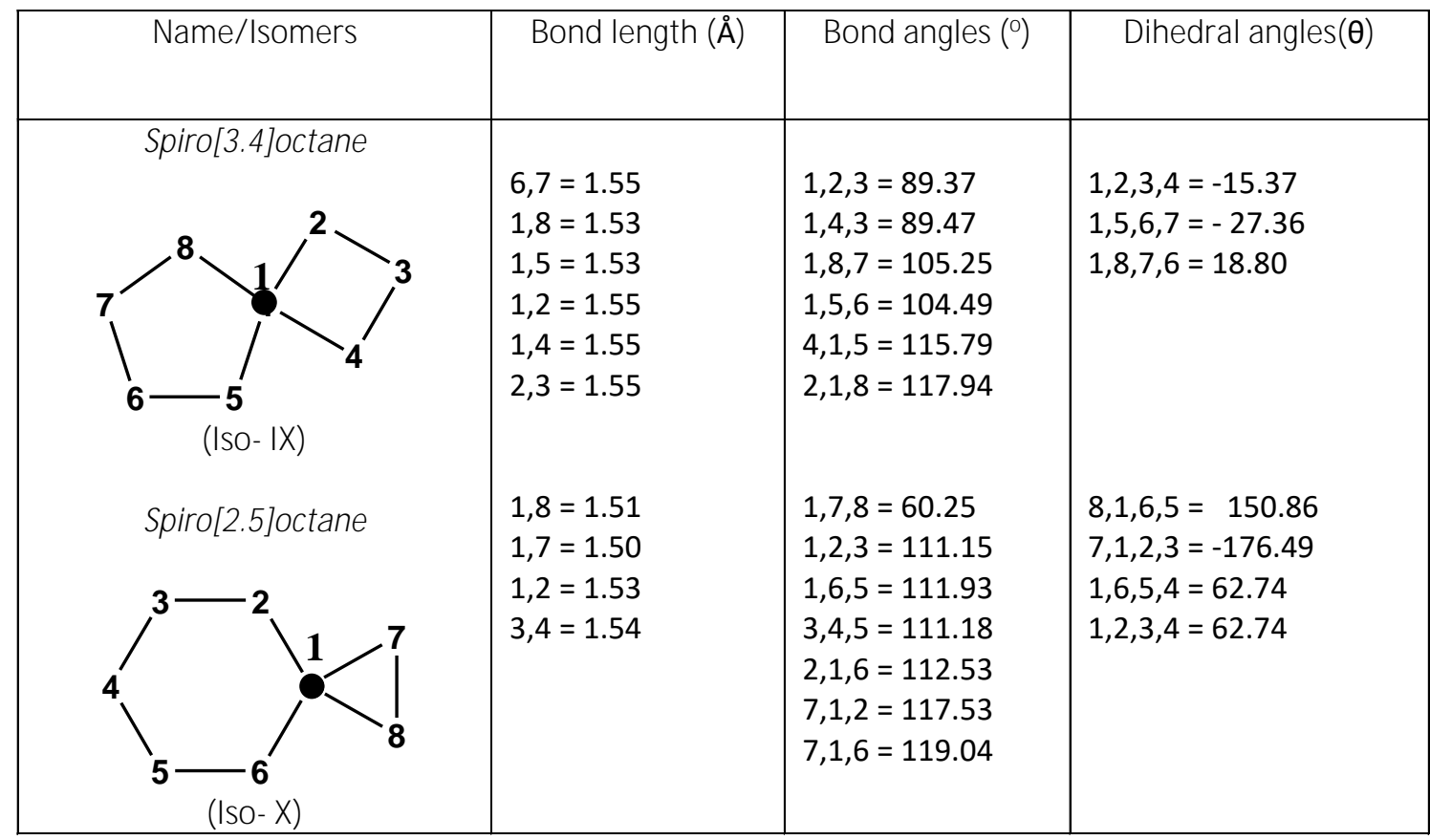

[C-1 \& C-2 of the figure in Table II(b)] to that of Iso-II, where C-1 \&C-4 [Figure in Table 2(a)] at the center are of normal tetrahedral angle range, ranging from $109.19^{\circ}$ to $109.71^{\circ}$. The difference in their enthalpy of formation is also only $0.46 \mathrm{kcals} / \mathrm{mol}$. Iso-III is a cis configurational isomer of Octahydropentalene with a dihedral angle $(\theta)$ of 122.10 cutting across the two center carbon atoms from the opposite ends of the two fused pentane rings as C3-C2-C1-C6 or C5-C1-C2-C8, thus without much angle strain coupled with a complete planar molecular moiety in both the pentane rings $(\theta=0.0)$. Iso-II on the other hand is a bridgedhead bicyclic ring of three equal 6membered ring sizes where part of each of the three 6 membered ring moieties projected out like a propeller (C5-C6 is taken as bridged head, along with C2-C3 and C7 -C8 [Figure in Table 2(a)]. All corresponding dihedral angles to the bridgedhead carbons (C1-C5-C6-C4 for C5-C6), C1-C2-C3-C4 for C2-C3 and C1-C8-C7-C4 for C7-C8 propeller projections are having respectively $\theta=3.78$ to their respective molecular place moiety, implying that there's a slight twist in the molecular moieties of each of the three 6-membered rings. The sum total twist of the molecular structure might be the contributing factor to the slight instability in comparison to Iso-III. The bond lengths for Iso-II and Iso-III are all within the range of C-C single bonds.

\section{Normal or fused bicyclic rings index}

All the corresponding geometrical indices for fused bicyclic rings are given in Table 2 (b). The counterpart of cis configurational isomer of octahydropentalene
(Iso-III) is the trans configurational isomer (Iso-IV), which is the next in its stability. In comparison to the cis configurational isomer, though we see that there's a decrease in the angle strain cutting across each of the two center carbon atoms fusing the two pentane rings $\left(106.79^{\circ}\right.$ to $\left.126.50^{\circ}\right)$ respectively, the dihedral angles passing through the two carbons fusing the two pentane rings ( $\theta=\mathrm{C} 8-\mathrm{C} 1-\mathrm{C} 5-\mathrm{C} 4$ and $\mathrm{C} 6-\mathrm{C} 5-\mathrm{C} 1-\mathrm{C} 2)$ are 180.00 and 179.09 respectively. This acquisition of planarity across the fused rings might have influenced the increase in angles between the two pentane rings at the point of fusion. Unlike the cis configurational isomer, the trans configurational isomer do not have planarity in the pentane moieties of the fused bicyclic rings. In fact, the dihedral angles show $\theta=14.14$ to 47.20 , twisting the molecular plane of each pentane moiety in and out of the plane of the molecule. All bond lengths are typical bond lengths of a C-C bond.

Among the fused bicyclic rings studied, we then have the configurational isomers of bicyclo[4.2.0]octane and bicyclo[5.1.0]octane both in the cis and trans configurational isomers. In both the cases the cis configurational isomers, i.e. cis bicyclo[4.2.0]octane (Iso-V) and cis bicyclo[5.1.0]octane (Iso-VII) are more stable than their corresponding counterparts trans configurational isomers. Bicyclo[4.2.0]octane is a fusion of 6-membered and 4-membered rings whereas bicyclo[5.1.0]octane is a fusion of 7-membered and 3-membered rings through two common carbon atoms bonding ( $1-\mathrm{C} 6$ for Iso- $\mathrm{V}$ and C1-C7 for Iso-VII). In both the cases of cis and trans configurational isomers, bicyclo[4.2.0]octane is more stable than the bicyclo[5.1.0]octane isomers. The reason may 
TABLE 2(d): Geometrical structures of isolated bicyclic rings showing the bond lengths $(\AA)$, bond angles $\left(^{\mathbf{0}}\right)$ and dihedral angles $(\theta)$.

\begin{tabular}{|c|c|c|c|}
\hline Name/ Isomers & Bond length $(\AA)$ & Bond angles $\left({ }^{\circ}\right)$ & Dihedral angles $(\theta)$ \\
\hline Trans-cyclopropylcyclopenpane & $\begin{array}{l}4,6=1.51 \\
6,7=1.51 \\
8,6=1.50 \\
7,8=1.50 \\
1,5=1.54 \\
4,3=1.54\end{array}$ & $\begin{array}{l}6,7,8=59.99 \\
3,4,5=102.26 \\
5,1,2=105.79 \\
1,2,3=105.78 \\
4,5,1=104.86 \\
4,3,2=104.85 \\
5,4,6=114.72 \\
8,6,4=120.99\end{array}$ & $\begin{array}{l}8,6,4,5=85.31 \\
8,6,4,3=156.68 \\
4,5,1,2=24.33 \\
4,3,2,1=-24.22 \\
9,6,4,10=-179.99 \\
9,6,4,3=-58.99 \\
9,6,4,5=59.00\end{array}$ \\
\hline Cis-cyclopropylcyclopenpane & $\begin{array}{l}1,6=1.51 \\
7,6=1.51 \\
8,6=1.50 \\
2,3=1.54 \\
5,4=1.55\end{array}$ & $\begin{array}{l}6,7,8=59.92 \\
2,1,5=102.57 \\
2,3,4=105.15 \\
1,5,4=105.90 \\
1,2,3=103.39 \\
2,1,6=118.13 \\
7,6,1=123.11\end{array}$ & $\begin{array}{l}1,2,3,4=35.05 \\
1,5,4,3=-10.83 \\
7,6,1,5=-81.06 \\
8,6,1,2=-34.19 \\
9,6,1,10=-57.01\end{array}$ \\
\hline $\begin{array}{l}\text { Cis-bicyclo butyl } \\
\qquad \stackrel{\mathrm{H}_{9}}{\equiv} \stackrel{\mathrm{H}_{10}}{\equiv} / \mathrm{S}\end{array}$ & $\begin{array}{l}1,5=1.52 \\
1,2=1.56 \\
1,4=1.55 \\
3,4=1.55 \\
5,8=1.55 \\
5,6=1.55\end{array}$ & $\begin{array}{l}1,2,3=88.75 \\
1,4,3=88.75 \\
5,8,7=88.75 \\
5,6,7=88.75 \\
8,5,1=117.45 \\
4,1,5=119.71\end{array}$ & $\begin{array}{l}1,2,3,4=5.35 \\
5,6,7,8=-17.93 \\
2,1,5,6=74.09 \\
4,1,5,6=-167.22 \\
9,1,5,10=-60.55\end{array}$ \\
\hline $\begin{array}{l}\text { Trans-bicyclo butyl } \\
\end{array}$ & $\begin{array}{l}1,5=1.52 \\
1,2=1.55 \\
1,4=1.55 \\
3,2=1.55 \\
3,4=1.55 \\
5,8=1.56 \\
5,6=1.56\end{array}$ & $\begin{array}{l}1,2,3=88.56 \\
1,4,3=88.67 \\
5,8,7=89.91 \\
5,6,7=89.87 \\
6,5,8=89.00 \\
8,5,1=119.33 \\
4,1,5=119.33\end{array}$ & $\begin{array}{l}5,8,7,6=17.93 \\
1,2,3,4=-17.93 \\
9,1,5,10=180.00\end{array}$ \\
\hline $\begin{array}{c}\text { Trans1,1-dimethyl bicyclopropyl } \\
\equiv\end{array}$ & $\begin{array}{l}1,4=1.52 \\
1,2=1.51 \\
1,3=1.51 \\
2,3=1.51 \\
4,5=1.51 \\
4,6=1.51 \\
4,8=1.52\end{array}$ & $\begin{array}{l}1,2,3=60.13 \\
4,5,6=60.13 \\
1,4,5=117.81 \\
1,4,6=119.82 \\
2,1,7=114.87 \\
6,4,8=115.61\end{array}$ & $\begin{array}{l}7,1,2,3=-106.36 \\
8,4,5,6=-106.33 \\
7,1,4,8=-161.10\end{array}$ \\
\hline
\end{tabular}

be due to the ring sizes with smaller ring size, and its contribution to ring strain and diaxial interactions in bicyclo[5.1.0]octane isomer. The cis isomers (Iso-V and IsoVII) occupy the fifth and the sixth stability order respectively, whereas the trans isomers are at the eleventh and the twelve positions. The less stability of Iso-VII is due to the strain with an angle of $60.32^{\circ}$ for the 3-membered ring moiety, whereas for Iso- $\mathrm{V}$ the angles $87.77^{\circ}$ and $88.11^{\circ}$ is a typical angle for cyclobutane, the 4- membered ring moiety. The bond angles ranging from $112.72^{\circ}$ to $122.24^{\circ}$ and $113.66^{\circ}$ to $123.38^{\circ}$ from the 6 membered ring and 7-membered ring moieties in Iso-V and Iso-VII, respectively all corresponds to the release in tetrahedral geometry. The dihedral angles in both the cases shows out of the plane twist of the molecule, where for Iso-VII with a larger ring moiety of 7membered encountered $\theta=47.68$ through one of the carbons fusing the two rings (C3-C2-C1-C8) and $\theta=-$ 
144.61on the other carbon atom (C5-C6-C7-C8). Moreover, the twist out of plane within the 7-membered ring moiety ranges from $\theta=77.01$ to 84.70 . This indicates that the twist within the system causes immense strain in the structure with three possible di-axial interactions. In Iso-V, lesser twisting across the fused rings and in the ring moieties is observed and the geometrical set up although it comes close to a stable chair conformation, it seems to either defy or decreased di-axial interactions encountered for substituted cyclohexane interactions [see dihedral angles in Table 2(b)].

The corresponding trans isomers on the other hand for bicyclo[4.2.0]octane and bicyclo[5.1.0]octane (Iso-VI and Iso-VIII, respectively) have bond angles ranging from $106.01^{\circ}$ to $131.74^{\circ}$ and $107.10^{\circ}$ to $132.62^{\circ}$, which can be considered as slightly more distorted bond angles for a 6 membered ring moiety and a 7-membered ring moiety. Bond angles of $86.67^{\circ}$ for the 4-membered ring moiety in Iso-VI and $59.43^{\circ}$ for the 3-membered ring moiety for Iso -VIII are as expected. The dihedral angles show the 4memberred ring moiety in $\mid$ so- $\mathrm{V} \mid$ to be puckered $(\theta=$ 39.65) and the twist out of plane for the 6-membered ring moiety $(\theta=52.24$ and 52.26$)$ for $\mathrm{C} 1-\mathrm{C} 2-\mathrm{C} 3-\mathrm{C} 4$ and $\mathrm{C} 6$ -C5-C4-C3, respectively entail a stable chair conformation but which seems to be off-set by the two di-axial interactions in both sides of the ring in rendering the trans configurational counterpart unstable by about $16.32 \mathrm{kcal} /$ mol. In Iso-VIII the dihedral angles indicate the orientations to increase the twist out plane as $(\theta=-27.13,81.69$ and 48.61) for C4-C5-C6-C7, C3-C4-C5-C6 and C1-C2-C3$\mathrm{C} 4$ respectively for the 7-membered ring moiety with possible four di-axial interactions, which may account for the less stability in comparison to Iso-VI by $12.11 \mathrm{kcal} /$ mol.

\section{Spiro bicyclic rings}

Table 2(c) represents the geometrical indexes for the spiro bicyclic rings where the stability order are the seventh and the eighth as Iso-IX > Iso- $X$, where Iso-IX is more stable by $1.96 \mathrm{kcals} / \mathrm{mol}$ than Iso-X. Iso-IX or spiro[3.4] octane is a bicyclic ring of 5-membered and 4-membered rings joint through a common carbon atom, and Iso-X or Spiro[2.5]Octane is that of a 6-membered and 3membered rings. The two ring moieties joint through a common carbon atom in spiro bicyclic rings are approximately perpendicular to each other is a known fact and can be seen from the dihedral angles. The instability of Iso- $X$ is due to the fact that the 6-membered ring moiety has a twist-boat conformer, aided with the strains encountered in the 3-membered ring moiety. On the other hand, the angles which come across the 5-membered ring moiety and the 4-membered ring moiety of Iso-IX are typical enveloped shaped cyclopentane and puckered cyclobutane perpendicular to each other which would cause less strain in comparison to the sum total strains experienced by the molecular plane. The data repre- sented in Table 2(c) fairly supports their interpretations as forms of distorted indexes for their geometrical structures.

\section{Isolated bicyclic rings}

The isolated bicyclic rings comprise of Iso-XI to Iso-XV and the stability order for this differentiation decreases to the least stability for the chosen system. The geometrical parameters are given in Table 2(d). Configurational isomers of fused bicyclic rings show more stability for the trans configurational isomers than the corresponding cis configurational isomers with the energies ranging from $22.82 \mathrm{kcal} / \mathrm{mol}$ to $45.21 \mathrm{kcal} / \mathrm{mol}(0.42 \mathrm{kcal} / \mathrm{mol}$ more than from Iso-X). The isolated bicyclic rings have an opposite resultant data for the conformational isomers with respect to fused bicyclic rings, where cis conformational isomers are more stable than the trans isomers. In fact, the corresponding trans conformational isomers for fused bicyclic rings, trans-bicyclo[4.2.0]octane (iso-vi) and trans-bicyclo[5.1.0]octane (Iso-VIII) falls within the energetic order of the isolated bicyclic rings in the bicyclic ring differentiation for the $\mathrm{C}_{8} \mathrm{H}_{14}$ system studied here with $26.16 \mathrm{kcal} / \mathrm{mol}$ and $32.02 \mathrm{kcal} / \mathrm{mol}$ for Iso-VI and Iso-VIII, respectively. In isolated bicyclic rings, the two ring moieties are joined through a $\mathrm{C}-\mathrm{C}$ single bond (as depicted in Figure 2). Trans-cyclopropylcyclopentane (Iso-XI) and cis-cyclopropylcyclopentane (Iso-XII) comprise of a 5-membered ring moiety and 3-membered ring moiety joint through a single $\mathrm{C}-\mathrm{C}$ bond. The enthalpies show that the trans conformational isomer is more stable than the cis conformational isomer. The stability of trans conformational isomer is as expected since the orientations of the ring moieties are symmetrically opposite to each other at the two ends of the $\mathrm{C}-\mathrm{C}$ bond, thus discouraging any forms of possible ring interactions. The value $\theta$ $=-179.99$ for $\mathrm{H} 9-\mathrm{C} 6-\mathrm{C} 4-\mathrm{H} 10$ indicates the two hydrogen atoms are opposite to each other and $\mathrm{H} 9$ lies exactly at the center of the cyclobutane ring with the dihedral angle as $\theta=58.99$ and 59.00 for H9-C6-C4-C3 and H9-C6-C4 $-\mathrm{C} 5$, respectively. Whereas, the conformational isomer has the ring moieties perpendicular to each other at the two ends of the $\mathrm{C}-\mathrm{C}$ bond with possible interactions between the ring moieties and thus rendering the isomer less stable. Dihedral angles of $\theta=-57.01$ for H9-C6-C1$\mathrm{H} 10$ indicates interactions between the hydrogen atoms to cause torsional strain and $\theta=-77.57$ and 62.07 for $\mathrm{H9}$ C6-C1-C2 and H9-C6-C1-C5, respectively also indicates the rotation about the $\mathrm{C}-\mathrm{C}$ sigma bond to bring about unwanted ring strains and with possible annihilation of the molecular symmetry. The 3-membered ring moiety in both the cases may also have relative contributions to its instability when considering cyclopropylcyclopentane isomers in positioning itself to be among the instable isomers for the system studied.

The corresponding analysis for the conformational isomers of bicyclo butyl isomers (Iso-XIII for trans and Iso 

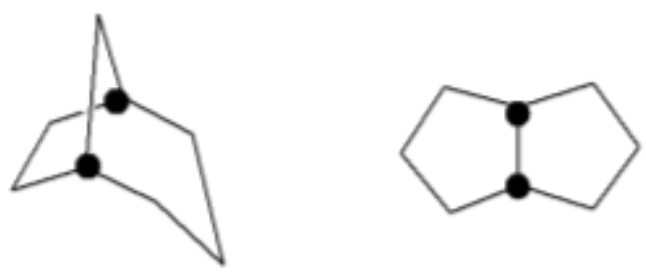

Bridgedhead bicyclic rings

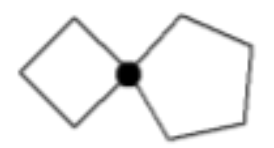

Spiro bicyclic Rings
Normal or Fused bicyclic rings

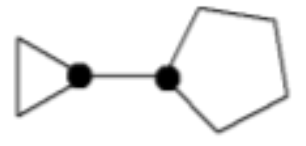

Isolated bicyclo rings
Figure 2: Bicyclic rings for the bridge head, the normal or fused, the spiro bicyclic rings and the isolated bicyclo rings. The highlighted black dots show the connection of the 2 rings in the atoms within the system, respectively.

-XV for cis) may also be rationalized similar to the explanations given above for cyclopropylcyclopentane conformational isomers. In Bicyclo butyl isomers, the two ring moieties are 4-membered rings connected by a C-C carbon atom bonds. The bond angles for both the conformational isomers (Iso-XIII for trans and Iso-XV for cis) ranges from $88.56^{\circ}$ to $89.00^{\circ}$, a typical angle range for cyclobutane. The less stability for the cis conformational isomer is due to the ring orientations across the molecular structure with the probability of ring interaction due to torsional strain as observed from $\theta=-60.55$ for H9-C1C5-H10 (For the trans counterpart $\theta=180.0$ ). Dihedral angles for $\mathrm{H} 9-\mathrm{C} 1-\mathrm{C} 5-\mathrm{C} 8$ and $\mathrm{H} 9-\mathrm{C} 1-\mathrm{C} 5-\mathrm{C} 6$ are 52.95 and 52.95 for Iso-XIII and 170.18 and 64.58 for Iso-XIV. The instability of bicyclo butyl isomers in comparison to cyclopropylcyclopentane isomers may be the angle strain of the 3-membered ring moiety in cyclopropylcyclopentane isomers is being off-set by the enveloped shaped 5membered ring Configurational isomers. In bicyclo butyl isomers, the two ring moieties are 4-membered rings connected by a C-C carbon atom bonds. The bond angles for both the conformational isomers (Iso-XIII for trans and Iso-XV for cis) ranges from $88.56^{\circ}$ to $89.00^{\circ}$, a typical angle range for cyclobutane. The less stability for the cis conformational isomer is due to the ring orientations across the molecular structure with the probability of ring interaction due to torsional strain as observed from $\theta=-60.55$ for H9-C1-C5-H10 (for the trans counterpart $\theta$ $=180.00)$. Dihedral angles for H9-C1-C5-C8 and H9-C1-C5 -C6 are 52.95 and --52.95 for Iso-XIII and 170.18 and $52.95 f o r$ Iso-XIV. The instability of bicyclo butyl isomers in comparison to cyclopropylcyclopentane isomers may be the angle strain of the 3-membered ring moiety in cyclopropylcyclopentane isomers is being off-set by the enveloped shaped 5-membered ring moiety. The difference in energy from Iso-XII (cis-cyclopropylcyclopentane) to Iso-XIII (trans-bicyclo butyl) is $14.35 \mathrm{kcal} / \mathrm{mol}$. This rather large difference in energy may be concluded with constrain to be the effect of ring sizes. The difference in energy between the conformational isomers of bicyclo butyl is only $0.88 \mathrm{kcal} / \mathrm{mol}$ in favor of the trans isomer.

Finally, we have the lease stable isomer, Iso-XV or trans-1,1-dimethyl bicyclopropyl isomer for our study here. This isolated bicyclic ring contains two 3-membered rings connected through a C-C bond. The bond angles for the two ring moieties are of a typical cyclopropane ring with $60.13^{\circ}$ for $\mathrm{C} 1-\mathrm{C} 2-\mathrm{C} 3$ and C4-C5-C6 as shown Table 2 (d). The bond angles incorporating the connecting C-C bond and the two methyl substituents with respect to the ring moieties have the angles ranging from $114.87^{\circ}$ to $119.82^{\circ}$ all bond angles close to tetrahedral. The dihedral angles between the two methyl groups and across the connecting $\mathrm{C}-\mathrm{C}$ bond is $\theta=-161.10$ (C7-C1-C4-C8) implying that the methyl groups as well as the two 3membered ring moieties are on the opposite side of the $\mathrm{C}-\mathrm{C}$ bond at the two ends. This isomer occupies the least stability due to the enormous angle strain manifested in the two 3-membered rings which would predict the fragile tendency to release the strains experienced by the molecular geometry. The dihedral angles $\theta=52.73$ (C7C4-C1-C3) and $\theta=-16.16$ (C7-C4-C1-C2) also indicates that the molecule is unsymmetrical with plausible torsional strains. The value of the bond lengths is all within $1.52 \AA$ and all the isomers studied for $\mathrm{C}_{8} \mathrm{H}_{14}$ system along with their configurational isomers correspond to a typical C-C bond of approximately $1.55 \AA$.

We may conclude that the geometrical analysis in demonstrating the parameters like the bond lengths $(\AA)$, bond angles $\left({ }^{\circ}\right)$ and dihedral angles $(\theta)$ relatively substantiate the resultant energetic data for the enthalpy of formations for the isomers studied. The energy diagram along the potential energy surface for the relative stability order of the fifteen isomers concluded earlier for $\mathrm{C}_{8} \mathrm{H}_{14}$ systems and calculated in B3LYP/6-311G (d) is shown Figure 3.

\section{Conclusion}

(1) The enthalpy of formation indicate a bridgedhead bicyclic ring, bicyclo[3.2.1]octane (Iso-I) to be the most stable and the isolated bicyclic ring, 1,1dimethylbicyclopropane (Iso-XV) to be the least stable. The stability order for all the isomers studied maybe given as, (Iso-I) $>$ (Iso-III) $>$ (Iso-II) $>$ (Iso-IV) $>$ (Iso-V) $>$ (Iso $-\mathrm{VII})>($ Iso-IX $)>($ Iso-X) $>$ (Iso-XI) $>$ (Iso-XII) $>$ (Iso-VI) $>$ (Iso $-\mathrm{VIII})>($ Iso-XIII) $>$ (Iso-XIV) $>$ (Iso-XV).

(2) The differentiation of the bicyclic rings into four categories relatively also follows the stability order as, bridged bicyclic rings $>$ normal or fused bicyclic rings $>$ spiro bicyclic rings $>$ Isolated bicyclic rings.

(3) The geometrical analysis relatively validates the stability order of the chosen isomers from the simulated resultant descriptions of the bond angles and the dihe- 


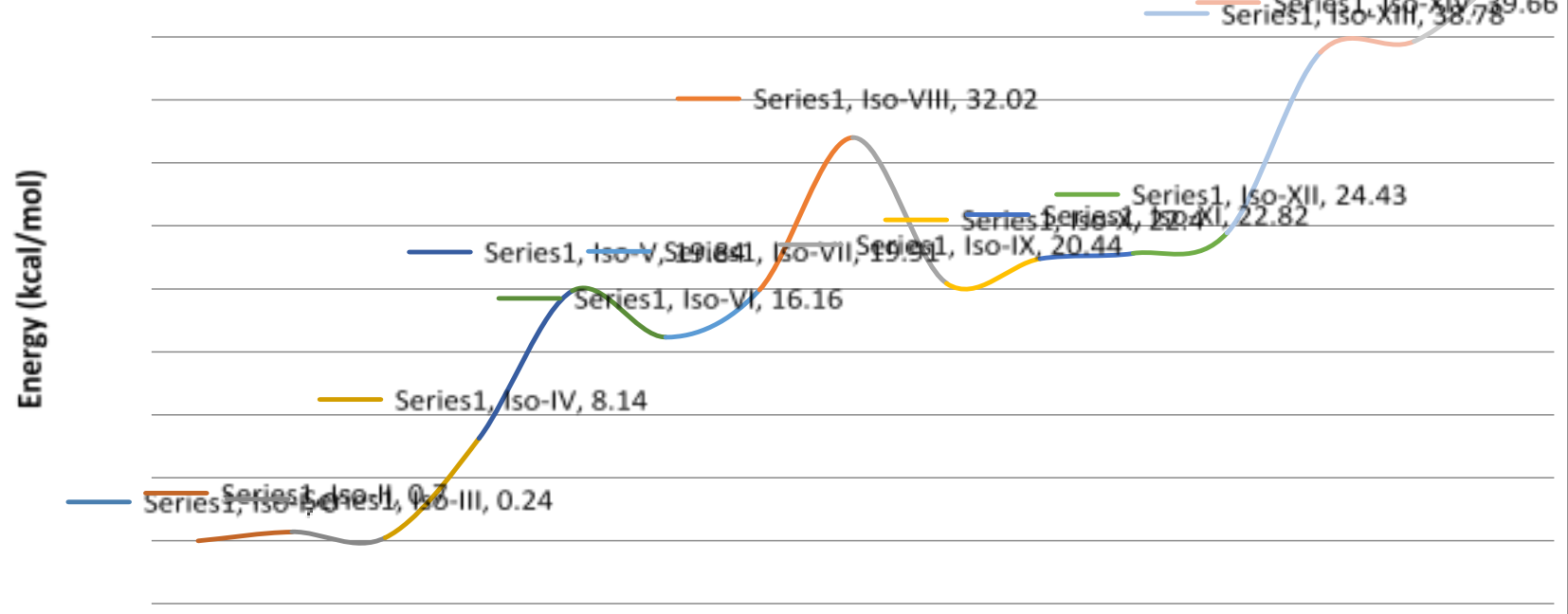

Figure 3: Energy diagram of $\mathrm{C}_{8} \mathrm{H}_{14}$ systems displaying ten isomers along with their configurational and conformational Isomers on the potential energy surface from B3LYP/6-311 $\mathrm{G}^{*}$ calculations.

dral angles. The configurational isomers amused the investigation by turning the leaf for stable cis configurational isomers within the fused bicyclic rings, in which nominally for a given configurational organic compounds, trans isomers are more stable in comparison. The reason may be the limiting levels of calculations in which the electronic orbitals are being approximated in a given space to affect the orientations the geometrical structures are founded upon. Moreover, configurational isomer entails fused ring structures with possible energetics from their ring conformations. Nevertheless, the isomers and their conformational isomers in isolated bicyclic rings do substantiate the trans conformational isomers to be more stable than their cis counterparts.

(4) Thermochemical analysis maybe included to gain in-depth understanding to their energetics, especially with the incorporation of zero-point vibrational energies (ZPVE) in usurping the total energy for further inquiry. Orbital approximations of split valence and increasing zeta polarized basis sets for the valence orbitals and its functions along with diffused functions may also be integrated. The geometrical arrangement in its comparative contribution to support the energetic may also find inquisition from higher theoretical concepts of electron correlations and orbital functional.

\section{REFERENCES}

Camps, P., Fernández, J., Vázquez, S. (2010). Synthesis of 1-substituted cis-bicyclo[3.3.0]octane-3,7-dione derivatives as potential precursors of polyquinanes. ARKIVOC 4, 74-89.

Lianbuanga, J., Pachuau, Z. (2011). Employment of ab initio of spiro, fused, bridged head and isolated rings of cyclo-octane isomers: Their relation with nature. Advances in Environmental Chemistry, 2011, 260264.

Kendhale, A., Gonnade, R., Rajamohanan, P., Sanjayan, G. (2008). A rigid bicyclo[3.3.0]octane (octahydropentalene): a heavily constrained novel aliphatic template for molecular self-assembly. Tetrahedron Letters, 49, 3056-3059.

Kozintsev, B. (1999). Gaussian User Manual. Gaussian Inc., U.S.A., pp. 10-30.

Kumar, H., (2008). A comparative QSPR study of alkanes with the help of computational chemistry. Bull Korean Chem. Soc. 29, 67-76.

Ma, C., Liu, T., Yang, L., Zu, Y., Chen, X., Zhang, L., Zhang, Y., Zhao, C. (2011). Ionic liquid-based microwaveassisted extraction of essential oil and biphenyl cyclooctenelignans from Schisandra chinensis Baill fruits. Journal of Chromatography A 1218, 8573-8580.

Prasad, R. (1996). Quantum Chemistry (4 ${ }^{\text {th }}$ revised edition). New Age International publication, New Delhi, pp. 50-200.

Watson, P., Coutsias, A., Thompson, A, Martin, S. (2010). Topology of cyclo-octane energy landscape. The Journal of Chemical Physics 132, 234115(1-6). 